\title{
Blends of Poly(3-Alkylthiophene) and [6,6]-Phenyl-C61-Butyric Acid Methyl Ester for Organic Photovoltaic Cell Applications: Multi-Scale Modeling Approach
}

\author{
Hanjong Yoo, ${ }^{1} \mathrm{Ki}$ Chul Kim,,${ }^{1, *}$ and Seung Soon Jang ${ }^{1,2,3, *}$ \\ ${ }^{1}$ Computational NanoBio Technology Laboratory, School of Materials Science and Engineering, \\ Georgia Institute of Technology, 771 Ferst Drive NW, Atlanta, Georgia 30332-0245, USA \\ ${ }^{2}$ Institute for Electronics and Nanotechnology, Georgia Institute of Technology, Atlanta, GA, \\ USA \\ ${ }^{3}$ Parker H. Petit Institute for Bioengineering and Bioscience, Georgia Institute of Technology, \\ Atlanta, GA, USA
}

\begin{abstract}
The interface-to-volume ratio of the structure and the transport property of the charge careers are the two main factors affecting the photovoltaic cell performance. In this study, the blends of poly(3-alkylthiophene) (P3AT) (A denotes H, N, and D for hexyl, nonyl, and dodecyl, respectively) and [6,6]-phenyl- $\mathrm{C}_{61}$-butyric acid methyl ester (PCBM) are investigated to understand the effect of the variation of molecular structure of the thiophene-based polymer in the active layer on the two main factors. First, we develop well-designed coarse-grained models to efficiently describe P3AT-PCBM systems. Next, the coarse-grained molecular dynamics simulations are performed to investigate the structural properties of three coarse-grained active layer systems, P3AT-PCBM. We conclude from our simulated analysis that the P3HT-PCBM system will provide the structurally optimized condition for the best photovoltaic cell performance. This is evidenced by two observations: (1) P3HT exhibits a good contact with PCBM in molecular level and (2) the P3HT-PCBM system forms a well-organized molecular network in each of the electron donor and accepter phases.
\end{abstract}


Keywords: Photovoltaic; Density Functional Theory; Molecular Dynamics Simulation; Force Field; Coarse-Grained Model 


\section{INTRODUCTION}

Harvesting the environmentally benign solar energy has been paid great amount of attention as one of the most promising approaches towards sustainable energy sources in order to resolve the current energy issues associated with the global warming and exhaustion of fossil fuels $[1,2]$. Although the silicon-based inorganic photovoltaic cell technology has made an important breakthrough for efficiently harvesting the solar energy [3], the cost of the devices currently impedes their practical use in a wide scope of applications. Hence, efforts to develop inexpensive energy harvesting devices have stimulated the introduction of new approaches based on relatively cheap organic materials. Indeed, a discovery of conjugated, semiconducting polymers such as poly(2-methoxy-5-(2'-ethyl-hexyloxy)-1,4-phenylene-vinylene) (MEH-PPV) have created a new class of promising solar cells, namely organic solar cells, which could potentially replace the inorganic photovoltaic cells [2, 4]. Yu et al. developed the first reported organic solar cells through blending MEH-PPV with fullerene (C60) and its derivatives [4]. Since then, their distinctive properties such as light-weight, mechanical flexibility, and processability in addition to the low cost have become the main attractive advantages of the organic photovoltaic cells with a great potential for clean energy production [5].

In such organic photovoltaic cells, the bulk-heterojunction (BHJ) morphology in the active layer has been accepted as the most well-understood model, which was first introduced by Yu et al.[4]. The BHJ is generally described as a nanophase-separated interpenetrating network of conjugated semiconducting polymers and electron accepting carbon materials such as fullerene derivatives and carbon nanotubes. Since it could be easily prepared in large areas by a single-step fabrication process such as solution-spincoating, spray-coating, dip-coating, and rollto-roll coating techniques [6-9], the BHJ has been widely studied and used.

To date, since the discovery of the MEH-PPV-C60 blend [4], various types of conjugated semiconducting polymers and carbon materials have been proposed as potential electron donoracceptor pair for improving cell performance. Currently, poly(3-hexylthiophene) (P3HT) and [6,6]-phenyl-C61-butyric acid methyl ester (PCBM) are regarded as a standard electron donoracceptor pair for the organic photovoltaic cell applications because 1) P3HT exhibits a higher hole mobility and broader range of the solar spectrum coverage than MEH-PPV and 2) PCBM has a higher solubility in organic solvents than C60 [10,11]. Despite their strengths, the organic photovoltaic cells based on the P3HT-PCBM blend still suffer from the relatively poor power 
conversion efficiencies $(\sim 12 \%)$ in comparison to the inorganic silicon photovoltaic cells $(\sim 25.6 \%)$ [12]. To overcome such poor power conversion efficiencies, a number of studies have been performed [13-19] by optimizing the morphology of the active layers [13-16]. For example, Heeger and coworkers reported that the postproduction with thermal annealing could improve the nanoscale morphology through increasing the crystallinity of P3HT, thereby facilitating the charge generation and transport [13]. Swinnen et al. employed various experimental techniques such as atomic force microscopy (AFM), transmission electron microscopy (TEM), and X-ray diffraction (XRD) to investigate the morphological properties of P3HT : PCBM blends in the active layer under various thermal conditions [14]. They verified that increasing the annealing temperature $\left(\geq 120^{\circ} \mathrm{C}\right)$ would impede the overall efficiencies of the organic photovoltaic cells by forming PCBM crystallites of undesired needle-like structures. Despite these experimental efforts, a comprehensive understanding on the optimal morphology within the active layer remains veiled, especially from the view of its relationship with molecular architectures of the materials.

The coarse-grained (CG) molecular dynamics (MD) simulation approach has been paid a special attention as a method providing meso-scale properties (beyond several tens of nanometer) for a past decade [20-23]. Indeed, the CG MD simulations have been extensively utilized to characterize the active layers [24, 25]. For example, To and Adams performed the CG MD simulations on three different models of P3HT-PCBM blends in order to investigate the correlation between the crystallinity of the P3HT-PCBM blends and the molecular orientation of the P3HT phase [24]. They observed that the crystallinity of the P3HT phase near the interfaces was quite dependent of the relative orientations of the $\mathrm{P} 3 \mathrm{HT}$ molecules with respect to the direction of the interfaces [24]. They concluded that the highest crystallinity of the P3HT phase was expected at the face-on orientation, meaning that the faces of thiophenes in the P3HT molecules were placed on the PCBM phase [24]. Lee et al. also performed CG MD simulations of P3HT-PCBM blends with various weight ratios to investigate the structures of the blends [25]. They analyzed interface-to-volume ratios for the models, domain sizes in each phase, and percolation ratios in each phase to analyze the mobility and available pathways of photo-induced charge carriers [25]. They concluded that the PCBM-P3HT blend model with the weight ratio of $1: 1$ was the optimal model with the largest interface-to-volume ratio and the balanced percolation [25]. 
In this work, we aim at developing new CG models to thoroughly describe the structural properties of the BHJ active layers in organic photovoltaic cells. Although the basic forms of the CG models were adopted from the models suggested by To and Adams [24] to represent poly(3alkylthiophene)s (P3ATs) (A = H, N, and D for hexyl, nonyl, and dodecyl, respectively) and PCBM molecules, we developed a force field defining the intra- and inter-molecular interactions

of the $\mathrm{CG}$ models on the basis of the corresponding full-atomistic models. The validity of this newly developed $\mathrm{CG}$ force field is confirmed by the reasonable agreement of the physical properties between simulations and experiments.

\section{MODELS AND SIMULATION METHODS}

\subsection{CG structural models}

P3ATs and PCBM are the main components forming the active layer in the organic photovoltaic cell. The detailed atomistic structures of the P3HT (the degree of polymerization $(\mathrm{DP})=2$ ) and PCBM, and their corresponding CG models are described in Fig. 1. Each repeating monomeric unit in a P3HT chain was mapped into three types of CG beads corresponding to the thiophene $\left(\mathrm{C}_{4} \mathrm{HS}\right), \mathrm{C}_{3} \mathrm{H}_{6}$, and $\mathrm{C}_{3} \mathrm{H}_{7}$ fragments at their centers of mass. Two methyl groups terminating the P3HT chain were assigned to two CG beads. Similarly, five types of CG beads were employed to assign $\mathrm{C}_{60}, \mathrm{C}_{2} \mathrm{H}_{2}, \mathrm{C}_{2} \mathrm{H}_{4}$, phenyl $\left(\mathrm{C}_{6} \mathrm{H}_{5}\right)$, and $\mathrm{COOCH}_{3}$ fragments in a PCBM. Each CG bead was positioned at its center of mass. After the CG bead generation, we set up simulated systems consisting of three different P3ATs (P3HT, P3NT, and P3DT) in the presence of PCBM with the initial density of $1.0 \mathrm{~g} / \mathrm{cm}^{3}$. The composition of each system was set to be $1: 1$ in weight for P3AT-PCBM blend. Each system contained 100 P3AT polymer chains with the DP of 50, while 916, 1160, and 1380 PCBM molecules formed an interface with P3HT, P3NT, and P3DT, respectively. It should be stressed that the full atomistic simulation model for the P3HT-PCBM system contained 125,000 atoms in the polymeric phase while corresponding CG simulation model only needed 15,000 beads in the polymeric phase. This infers that the CG models can cover large-scale simulations in terms of size and time.

\subsection{CG force field development}

In order to use the aforementioned CG models, the corresponding CG force field should also be developed. Please note that force field (FF) is a collection of potential energy functions and related parameters, which evaluates the interaction energies of the structures. Here, we 
performed two-step process to develop the CG FF. In step 1, a full-atomistic FF was developed using the quantum mechanical density functional theory (DFT) method. This full-atomistic FF is used as a reference FF in developing a CG FF. In step 2, the CG FF parameters were determined based on the full-atomistic FF developed in step 1.

Step 1. To develop a CG FF, we need to start from an accurate full-atomistic FF. For this, we employed the DREIDING FF which is widely-used generic full-atomistic FF for various material systems [26-31]. To describe the molecular interactions for the P3AT-P3AT pair and the PCBM-PCBM pair, we used the DREIDING FF without adjustment owing to its reasonable accuracy. In contrast, we optimized parameters in the DREIDING FF for the interaction between P3AT and PCBM, particularly, the interaction between thiophene (of P3AT) and C60 (of PCBM), since there was no report on the reliability of the DREIDING FF parameters in evaluating the interaction energies in comparison to those obtained from DFT calculations.

Therefore, first, we characterized the energy values as a function of distance between P3AT and PCBM using DFT-D3, a dispersion-corrected DFT method [32, 33] with general gradient approximation (GGA) Perdew-Burke-Ernzerhof (PBE) functional [34] in Jaguar [35]. For this task, three configurations, namely a thiophene placed on a six-membered ring (Fig. 2a), five-membered ring (Fig. 2b), and a bond between hexagon and pentagon of a C60 (Fig. 2c), are used. Here, it is worthwhile to note that a CG model for the thiophene-C60 pair where a C60 fragment is replaced with one bead would not distinguish such detailed configurations.

Second, the full-atomistic FF was fit to reproduce the DFT-D3 based interaction energies between P3AT and PCBM. The potential energy functions and parameters for the full-atomistic thiophene-C60 pair are defined by the following equation:

$V=\sum_{\text {bonds }} \frac{1}{2} k_{\text {bond }}\left(r-r_{e}\right)^{2}+\sum_{\text {angles }} \frac{1}{2} k_{\text {angle }}\left(\theta-\theta_{0}\right)^{2}+\sum_{\text {torsions }} \frac{1}{2} k_{\text {torsion }}(1+$ $\left.\cos \left(n \varphi-\varphi_{0}\right)\right)+\sum_{\text {coulomb }}^{i<j} \frac{q_{i} q_{j}}{r_{i j}}+\sum_{v d W} D_{e}\left(1-e^{-a\left(r-r_{e}\right)}\right)^{2}$

where $V$ is the potential energy, $k_{\text {bond }}, k_{\text {angle }}$, and $k_{\text {torsion }}$ are force constants in bond, angle and torsion, respectively, and $r_{e}, \theta_{0}$, and $\varphi_{0}$ are the equilibrium length, equilibrium angle, and equilibrium torsion angle, respectively. $n$ denotes periodicity, $q_{i}$ and $q_{j}$ are atomic charges in 
electron units, and $r_{i j}$ is the distance between $i$ and $j$ atoms. Note that the Morse potential function is employed to describe the pairwise van der Waals interactions. In general, the Morse potential with three parameters is computationally more expensive but more accurate in describing the short-distance interaction in comparison to the Lennard-Jones potential with two parameters. The atomic charges are assigned using Mulliken population analysis.

Step 2. The interaction energies for three pairs, namely P3AT-P3AT, PCBM-PCBM, and P3AT-PCBM, were calculated using the full-atomistic FF functions and parameters to determine the CG FF functions and parameters. Then, the corresponding CG structures of the three pairs were then constructed as described above. Finally, the CG FF was applied to the CG structures and optimized to reproduce the full-atomistic interaction energies. The complete FF functions and parameters employed for the corresponding CG structures are summarized in the Supporting Information (Tables S1-S6).

\subsection{MD simulation approach}

CG MD simulations with the newly developed CG FF were performed using the Large-scale Atomic/Molecular Massively Parallel Simulator (LAMMPS) package [36] to investigate the physical and structural properties of the systems consisting of electron donors (P3HT, P3NT, and P3DT) and acceptors (PCBM). The equation of motion was integrated using the Verlet algorithm with a time step of $1.0 \mathrm{fs}$ [37]. To accelerate the equilibration process, the annealing simulations were performed through the following steps: 1) a simulated system was gradually expanded with increasing temperature from $300 \mathrm{~K}$ to $500 \mathrm{~K}$ for $100 \mathrm{ps}$; 2) the NVT MD simulation was performed at $500 \mathrm{~K}$ for $200 \mathrm{ps;}$ 3) the system was compressed to have a target density with decreasing temperature from $500 \mathrm{~K}$ to $300 \mathrm{~K}$ for $100 \mathrm{ps}$. This procedure was repeated five times to accelerate the equilibration. The annealing simulation was finalized by performing a NVT MD simulation for $2 \mathrm{~ns}$ at $298 \mathrm{~K}$. To collect the data, we performed an extended NPT MD simulation for $30 \mathrm{~ns}$ at $298 \mathrm{~K}$ as production MD simulation. To control the temperature, the Nosè-Hoover thermostat with a dimensionless cell mass factor of 1.0 was used [38-40]. It was confirmed that the simulation cell sizes of the P3HT-PCBM, P3NT-PCBM, and P3DT-PCBM CG models were converged to the cell dimensions of $13.4,14.1$, and $15.3 \mathrm{~nm}$, respectively, during the NPT MD simulations.

\section{RESULTS AND DISCUSSION}

\subsection{Validation of CG MD force field}


In contrast to the full atomistic model, the CG model is composed with a few number of beads representing many atoms as shown in Fig. 1, enabling the simulation to describe larger systems with a longer time scale [41, 42]. Despite the advantage of the CG models to overcome the limitation of the full atomistic models, the accuracy of the CG model should be clearly verified to draw confident conclusions from the simulations. In this section, our CG models on P3HT and PCBM are validated in two different aspects: pair interaction energy and physical properties.

In the pair interaction energy, the quality of the CG FF for reproducing the interaction energy of the three full-atomistic pairs, namely P3AT-P3AT, PCBM-PCBM, and P3AT-PCBM, has to be verified. As presented in the Figs. 3, 4, and 5, the interaction energies calculated from our CG models with the CG FF have a good agreement with those from the full-atomistic counterparts.

The next validation was considered by calculating physical properties of the system such as the densities and solubility parameters of P3HT and PCBM systems. We first prepared for two full-atomistic systems, each of which contains 10 P3HT chains with the DP of 50 and 100 PCBM molecules. Then, their CG counterparts were also prepared with the same sizes for comparison. First, the aforementioned annealing simulation was performed to accelerate the equilibration of the systems, and then, the physical properties of the systems were characterized through the production MD simulations.

The DREIDING FF was employed for the full atomistic MD simulations while the newly developed CG FF was used in the CG MD simulations. The equilibrated structures for P3HTPCBM, P3NT-PCBM, and P3DT-PCBM CG models are shown in Fig. 6. The detailed analyses of the physical properties of the P3HT and PCBM systems are described below for the validation of the CG FF.

The density predicted by the full-atomistic MD simulations for each of the P3HT and PCBM systems is listed in Table 1 in comparison with the reported experimental density. The simulated densities of CG models are $0.822 \mathrm{~g} / \mathrm{cm}^{3}$ and $1.47 \mathrm{~g} / \mathrm{cm}^{3}$ for the P3HT and PCBM, respectively. Compared to the reported experimental densities are $0.936 \mathrm{~g} / \mathrm{cm}^{3}$ and $1.50 \mathrm{~g} / \mathrm{cm}^{3}$ for the P3HT and PCBM, respectively, the densities from CG models have even better agreement with the experimental values than the densities from full-atomistic model $\left(0.710 \mathrm{~g} / \mathrm{cm}^{3}\right.$ and 1.46 $\mathrm{g} / \mathrm{cm}^{3}$ for the P3HT and PCBM, respectively). The poor results of the density of P3HT from the full-atomistic model indicates that the Dreiding FF is not fully optimized for the P3HT system. 
However, it is still noted in Table 1 that the density of P3HT in CG model is smaller than the experimental density. Since a general consensus explaining why the experimental densities are normally larger than the simulated ones is that the experimental samples would always include crystalline phase of larger density, we think that the underestimation of the densities from the P3HT model would be attributed to the structural difference between experiment and simulated system [43].

The second physical property, namely solubility parameter, was also calculated to check the accuracy of the CG FF, which could be crucial for the miscibility behaviors. The solubility parameter of a material is defined as the squared root form of the cohesive energy density as described in Eq. (2):

$$
\delta=\sqrt{\frac{\Delta H-R T}{V}}
$$

where $\Delta H$ denotes the enthalpy of vaporization, $R$ and $T$ denote the gas constant and absolute temperature, and $V$ is the system volume in equilibrium state. The solubility parameters calculated using the full-atomistic and CG FFs are presented in Table 2 in comparison with the corresponding experimental values, showing that the simulated solubility parameters (11.7 for P3HT and 5.7 for PCBM) of P3HT and PCBM are reasonably agreed with the experimental solubility parameters. It is again confirmed that the CG value is closer to the experimental one in comparison with the full-atomistic value. However, the solubility parameters of CG model needs more improvement. We think this is due to the lack of configurational diversity in our simulation.

Summarizing the validation, it is confirmed that the CG models with the newly developed CG FF can describe 1) the interaction energies of the P3AT-P3AT, PCBM-PCBM, and P3ATPCBM pairs, and 2) the physical properties, namely density and solubility parameter, of the P3HT and PCBM phases. All these suggest that the CG models and CF FF developed in this study are applicable to further investigations.

\subsection{Structural properties of P3AT-PCBM systems}

It has been understood that the power conversion efficiency of organic photovoltaic cell depends on the three main mechanisms, namely the photo-induced exciton generation, the dissociation of the generated excitons, and the transport of the separated charge carriers toward electrodes. Therefore, it is very important to design desirable nanoscale structure of the active layer facilitating the three main mechanisms for the high efficiency: the photo-induced Coulomb 
bound excitons are generated on the electron donor - acceptor interface, so that the number of the excitons would be increased as a function of the interface-to-volume ratio, while the dissociation of the excitons and the transport of the charge carriers can be facilitated by the appropriate molecular arrangement of the electron donor and acceptor. Thus, we employed the CG MD simulation approach with the validated CG FF to investigate the structural properties of the active layers consisting of P3AT (the electron donor) and PCBM (the electron acceptor). Please note that we focused only on the structures in this study without probing the electrochemical properties such as generation and dissociation of excitons and charge transport because the classical MD simulation method do not provide relevant information for such electrochemical properties.

Interface-to-volume ratio. To characterize the interface-to-volume ratios of the three CG models of P3AT-PCBM systems $(\mathrm{A}=\mathrm{H}, \mathrm{N}$, or D for hexyl, nonyl, and dodecyl, respectively), we employed the Connolly surface analysis in which the surface area of a target system is evaluated using a probing particle rolling over the target $[44,45]$. For this, we first removed the P3AT from the systems and then implemented the Connolly surface analysis by rolling a probe with the radius of $5.5 \AA$ over the PCBM phase. Here, the calculated surface area of the PCBM phase is considered as the interfacial area between the P3AT and PCBM phases. Note that the probe radius of $5.5 \AA$ is the van der Waals radius of C60. The interface-to-volume ratio is calculated by the following equation:

$$
\gamma=A_{\text {interface }} / V_{\text {box }},
$$

where $A_{\text {interface }}$ is the total interfacial area and $V_{b o x}$ is the volume of the simulation box. To obtain the two terms in Eq. (3), we performed the NPT MD simulations for $50 \mathrm{~ns}$ and then the last $10 \mathrm{~ns}$ showing the equilibrium state was chosen for the analysis. The change in the interface-to-volume ratio over the time is shown in Fig. 7. It is revealed that the interface-to-volume ratio is increased with increasing the side chain length in the P3AT: 1.13, 1.16, and 1.23 for P3HT-PCBM, P3NTPCBM, and P3DT-PCBM, respectively. This indicates that increasing the side chain length of the P3AT improves the miscibility with the functional group in the PCBM, which has been known to lead to the increase of the number density of the photo-generated excitons.

Nanophase arrangement of electron donor and acceptor. To investigate the spatial distribution of the components in the active layers, we analyzed the pair correlation functions (PCFs), defined by time-averaged probabilities of finding particles at a distance $r$ from a central 
particle. From our simulations, the PCFs of the three pairs such as thiophene-thiophene, C60C60, and C60-thiophene pairs, were calculated. The PCFs for the thiophene-thiophene pair and C60-C60 pair could provide clues in understanding how the hole and electron could transport through the active layers, while the PCF for the C60-thiophene pair would provide a clue for the dissociation of the excitons at the electron donor - acceptor interface. The PCF is calculated by

$$
g_{A-B}(r)=\left(\frac{n_{A-B}}{4 \pi r^{2} \Delta r}\right) / \frac{N_{B}}{V}
$$

where $n_{A-B}$ is the number of $B$ particles found within the shell of thickness $\Delta r$ from a particle, $A$, and $N_{B}$ and $V$ are the total number of the $B$ particles in the system and the total volume, respectively. The $A$ and $B$ can be either thiophene or C60. To count the number of $\mathrm{B}$ around A, the $g(r)$ is multiplied by the total number density $\left(\rho=N_{B} / V\right)$ of the $B$ particles in the system as shown in Fig. 8. For a quantitative analysis, the coordination number $(\mathrm{CN})$ of $\mathrm{B}$ for $\mathrm{A}$ (i.e., the number of B around A) is also calculated by integrating the first solvation shell of the PCF as listed in Table 3.

The main observation from the number density-weighted PCF for the thiophenethiophene pair for each of the CG P3AT-PCBM systems shown in Fig. 8a is that peaks are regularly placed with the same interval $(3.9 \AA)$ as a function of the distance. The peaks are originated from the distributions of the thiophene units within the intrachains of the P3AT, so that the peak positions have the same values regardless of the side chain length. The strongest peak placed at $3.9 \AA$ corresponds to the nearest neighboring thiophene along the chain. In the same logic, the second and third strong peaks at $7.8 \AA$ and $11.7 \AA$ are assigned to the second and third nearest neighboring thiophene pairs, respectively. It is worthwhile to note that the number density-weighted PCF intensity in Fig. 8a consistently follows the order of P3HT > P3NT > P3DT at the entire range of the distance although the difference is very small, which is consistent with the value of $\mathrm{CN}$ reported in Table 3. Given that all three systems have the same number of chains with the same DP, the difference in the intensity among the three systems arises from the difference in the spatial distribution among the P3AT polymer chains.

The number density-weighted PCF for the C60-C60 pair describes that the PCBM molecules in each system tend to have the distances of $\sim 10 \AA$ with other PCBM, indicating that the C60 faces another C60 directly. On the other hand, another peak observed at the distances of $\sim 13 \AA$ indicates that two PCBM contacts through the butyric acid methyl ester groups. It is important to note that electrons are transported through the C60 fragments. In that sense, a CG 
P3HT-PCBM system exhibiting that the stronger correlation of C60-C60 could be better condition to achieve the optimal structures in the active layer for the electron mobility. The CN in Table 3 infers the same conclusion. In other words, the CG P3HT-PCBM system shows the highest $\mathrm{CN}$ for the $\mathrm{C} 60-\mathrm{C} 60$ pair compared to the other two systems, indicating that the P3HTPCBM system allows the highest aggregation of the C60s.

As discussed above, we think that the number density-weighted PCFs for the thiophenethiophene and C60-C60 pairs could be related to the hole and electron mobilities through the electron donor and acceptor phases within the active layer. Similarly, the number densityweighted PCFs for the C60-thiophene pair shown in Fig. 8c could be related to the dissociation of the excitons at the interface between P3AT phase and PCBM phase. In molecular level, it is believed that the probability for the exciton dissociation is enhanced as a function of the molecular contact between P3AT (thiophene fragment) and PCBM (C60 fragment). The CN for each system is calculated as listed in Table 3. The strongest PCF for the C60-thiophene pair at the first peak is found from the interface of the P3HT-PCBM system, leading to its highest CN (5.23) as compared with the other two systems. This infers that the P3HT-PCBM system would form an optimal structure at the interface for the exciton dissociation.

Evaluation of the ability of the three CG active layers: The two factors mentioned above could be related to the exciton generation, exciton dissociation, and charge transport for the three CG active layers. For instance, the first factor represents the interface-to-volume ratio for indirectly assessing the exciton generation while the second factor contains three number density-weighted PCFs related to the exciton dissociation and charge transport. One might also think of the possibility for evaluating the three main mechanisms together. Here, the exciton flow parameter, $P_{\text {exciton}}$, is calculated to comprehensively describe the three main mechanisms by simply multiplying the two factors, as defined by

$$
P_{\text {exciton }}=K\left(\gamma \times C N_{C 60-\text { thiophene }}\right)
$$

where $K$ is the constant describing the number of the C60 per unit area, $\gamma$ is the interface-tovolume ratio over the simulation time, and $C N_{C 60-\text { thiophene }}$ is the coordination number of thiophene fragments for a C60. The exciton flow parameter describes the total number of the thiophene geometrically coordinating all the C60 placed at the electron donor-acceptor interface. This parameter provide a rough idea on how many electrons can be transferred from thiophene to C60. Fig. 9 shows the change of the calculated exciton flow parameters as a function of the 
simulation time for the three CG P3AT-PCBM systems. It is clearly demonstrated that the P3HT-PCBM system has the highest value in the exciton flow parameter in comparison to the other two CG systems, suggesting that the CG P3HT-PCBM system forms the interface through which the greatest number of the electrons could transport. This agrees well with the experimental observation reported by Cho et al. [46].

Considering the two factors, namely the interface-to-volume ratio and nanophase arrangement of electron donor and acceptor, the CG P3HT-PCBM system is inferred to provide the optimal structures for the best performance among the three systems owing to the two reasons. First, it is found that the CG P3HT-PCBM system has considerably better contact between the electron donor phase and acceptor phase in molecular level in comparison to the other two systems. This better contact between the phases overcompensates for the negative effect of the relatively poor interface-to-volume ratio of the P3HT-PCBM system compared to the other two systems. Second, it is revealed that the P3HT-PCBM system attains better molecular network consisting of the electron donor and accepter phases. This facilitates the charge carrier transport through the phases toward the electrodes. All these suggest that the performance of the organic photovoltaic cell depends not only on the interfacial area but also on the molecular network in the active layer.

According to our study, we may expect that the shorter side chain in P3AT would may improve the charge transport efficiency by forming better interface through the molecular interaction with PCBM. Thus, we better scrutinize this possibility in future study.

\section{Conclusions}

We developed coarse-grained (CG) models with CG force field (FF) for the P3AT-PCBM systems ( $\mathrm{A}=\mathrm{H}, \mathrm{N}$, and $\mathrm{D}$ for hexyl, nonyl, and dodecyl, respectively) which could be applied to simulate organic photovoltaic cells. The CG FF was validated to describe the interaction energies between components in the active layer systems. The developed CG FF was further confirmed to reproduce the physical properties such as density and solubility parameter of the P3AT and PCBM systems.

The CG molecular dynamics (MD) simulations using the developed CG FF were performed to investigate the effect of the side chain length of the electron donor (P3AT) on the structure of the active layer. In this study, the alkyl side chain length of the thiophene-based 
polymer was varied from the hexyl to the dodecyl. From our CG MD simulations investigating the structures of active layer systems composed of P3AT and PCBM, we found that the P3HTPCBM system would have more desirable structural features for performance, which was in a good agreement with experimental observations [47-49]. One main conclusion dawn from the simulations was that the CG P3HT-PCBM system would have an optimal structure attaining good features: 1) the fairly good contact between P3HT and PCBM in molecular level in comparison with P3NT and P3DT and 2) the CG P3HT-PCBM system with better molecular network consisting of the electron donor and accepter phases in comparison with the other two cases.

\section{ASSOCIATED CONTENT}

Details of the force field parameters and fitted potential energy surfaces. Model S1. Tables S1S6.

\section{AUTHOR INFORMATION}

${ }^{[*]}$ Corresponding Authors.

E-mail:kichulk@gatech.edu; seungsoon.jang@mse.gatech.edu

\section{AUTHOR CONTRIBUTIONS}

The manuscript was written through contributions of all authors. All authors have given approval to the final version of the manuscript.

\section{ACKNOWLEDGMENT}

We acknowledge that this research used resources of the Keeneland Computing Facility at the Georgia Institute of Technology, supported by the National Science Foundation under Contract OCI-0910735.

\section{REFERENCES}

[1] L. Lu, T. Zheng, Q. Wu, A.M. Schneider, D. Zhao, L. Yu, Chem. Rev., 115 (2015) 1266612731.

[2] G. Li, R. Zhu, Y. Yang, Nature Photon., 6 (2012) 153-161.

[3] Y. Lee, C. Park, N. Balaji, Y.-J. Lee, V.A. Dao, Isr. J. Chem. , 55 (2015) 1050-1063.

[4] G. Yu, J. Gao, J.C. Hummelen, F. Wudl, A.J. Heeger, Science, 270 (1995) 1789-1791.

[5] K.A. Mazzio, C.K. Luscombe, Chem. Soc. Rev., 44 (2015) 78-90. 
[6] A. Reale, L.L.N.L. Salamandra, G. Polino, G. Susanna, T.M. Brown, F. Brunetti, A.D. Carlo, Energy Technol., 3 (2015) 385-406.

[7] M. Eslamian, Coatings, 4 (2014) 60-84.

[8] M. Eslamian, F. Zabihi, Nanoscale Res. Lett., 10 (2015) 462.

[9] F. Yan, J. Noble, J. Peltola, S. Wicks, S. Balasubramanian, Sol. Energy Mater. Sol. Cells, 114 (2013) 214-218.

[10] M.T. Dang, L. Hirsch, G. Wantz, Adv. Mater., 23 (2011) 3597-3602.

[11] K.C. Kim, J.H. Park, O.O. Park, Sol. Energy Mater. Sol. Cells, 92 (2008) 1188-1191.

[12] K. Bullis, in, MIT Technology Review, 2014.

[13] W. Ma, C. Yang, X. Gong, K. Lee, A.J. Heeger, Adv. Func. Mater., 15 (2005) 1617-1622.

[14] A. Swinnen, I. Haeldermans, P. Vanlaeke, J. D'Haen, J. Poortmans, M. D'Olieslaeger, J.V. Manca, Eur. Phys. J-Appl. Phys., 36 (2006) 251-256.

[15] C. Du, Y. Ji, J. Xue, T. Hou, J. Tang, S.-T. Lee, Y. Li, Sci. Rep., 5 (2015) 16854.

[16] A. Kumar, G. Li, Z. Hong, Y. Yang, Nanotechnology, 20 (2009) 165202.

[17] H.-Y. Chen, J. Hou, S. Zhang, Y. Liang, G. Yang, Y. Yang, L. Yu, Y. Wu, G. Li, Nature Photon., 3 (2009) 649-653.

[18] Z. He, C. Zhong, S. Su, M. Xu, H. Wu, Y. Cao, Nature Photon., 6 (2012) 591-595.

[19] G. Zhao, Y. He, Y. Li, Adv. Mater., 22 (2010) 4355-4358.

[20] A.Y. Shih, A. Arkhipov, P.L. Freddolino, K. Schulten, J. Phys. Chem. B, 110 (2006) 36743684.

[21] A.Y. Shih, P.L. Freddolino, A. Arkhipov, K. Schulten, J. Struct. Biol., 157 (2007) 579-592.

[22] A. Arkhipov, P.L. Freddolino, K. Schulten, Structure, 14 (2006) 1767-1777.

[23] A. Arkhipov, Y. Yin, K. Schulten, Biophys. J., 95 (2008) 2806-2821.

[24] T.T. To, S. Adams, Phys. Chem. Chem. Phys., 16 (2014) 4653-4663.

[25] C.-K. Lee, C.-W. Pao, C.-W. Chu, Energy Environ. Sci., 4 (2011) 4124-4132.

[26] K.C. Kim, E.G. Moschetta, C.W. Jones, S.S. Jang, J. Am. Chem. Soc., 138 (2016) 76647672.

[27] G. Brunello, S.G. Lee, S.S. Jang, Y. Qi, J. Renewable Sustainable Energy, 1 (2009) 033101.

[28] Y. Chang, G.F. Brunello, J. Fuller, M. Hawley, Y.S. Kim, M. Disabb-Miller, M.A. Hickner, S.S. Jang, C. Bae, Macromolecules, 44 (2011) 8458-8469.

[29] S.G. Lee, G.F. Brunello, S.S. Jang, J.H. Lee, D.G. Bucknall, J. Phys. Chem. B, 113 (2009) 6604-6612.

[30] K.W. Han, K.H. Ko, K. Abu-Hakmeh, C. Bae, Y.J. Sohn, S.S. Jang, J. Phys. Chem. C, 118 (2014) 12577-12587.

[31] S.D. Snow, K.C. Kim, K.J. Moor, S.S. Jang, J.-H. Kim, Environ. Sci. Technol., 49 (2015) 2147-2155.

[32] S. Grimme, J. Antony, S. Ehrlich, H. Krieg, J. Chem. Phys., 132 (2010) 154104.

[33] S. Kwon, J.I. Choi, S.G. Lee, S.S. Jang, Comput. Mater. Sci., 95 (2014) 181-186.

[34] J.P. Perdew, K. Burke, M. Ernzerhof, Phys. Rev. Lett., 77 (1996) 3865-3868.

[35] in, Schrodinger, LLC, New York, 2009.

[36] S. Plimpton, J. Comput. Phys., 117 (1995) 1-19.

[37] L. Verlet, Phys. Rev., 159 (1967) 98-103.

[38] S. Nosé, J. Chem. Phys., 81 (1984) 511-519.

[39] S. Nosé, Mol. Phys., 52 (1984) 255-268.

[40] S. Nosé, Mol. Phys., 57 (1986) 187-191.

[41] R.K.Z. Tan, A.S. Petrov, S.C. Harvey, J. Chem. Theory Comput., 2 (2005) 529-540. 
[42] M. Neri, C. Anselmi, M. Cascella, A. Maritan, P. Carloni, Phys. Rev. Lett., 95 (2005) 218102.

[43] D. Pavel, R. Shanks, Polymer, 44 (2003) 6713-6724.

[44] in, Accelrys, Inc., San Diego, 2014.

[45] T. Düren, F. Millange, G. Ferey, K.S. Walton, R.Q. Snurr, J. Phys. Chem. C, 111 (2007) $15350-15356$.

[46] C.-H. Cho, H.J. Kim, H. Kang, T.J. Shin, B.J. Kim, J. Mater. Chem., 22 (2012) 1423614245.

[47] W.Y. Huang, C.C. Lee, S.G. Wang, Y.K. Han, M.Y. Chang, J. Electrochem. Soc., 157 (2010) B1336-B1342.

[48] L.H. Nguyen, H. Hoppe, T. Erb, S. Günes, G. Gobsch, N.S. Sariciftci, Adv. Func. Mater., 17 (2007) 1071-1078.

[49] A. Gadisa, W.D. Oosterbaan, K. Vandewal, J.-C. Bolsee, S. Bertho, J. D'Haen, L. Lutsen, D. Vanderzande, J.V. Manca, Adv. Func. Mater., 19 (2009) 3300-3306.

[50] C.W.T. Bulle-Lieuwma, W.J.H.v. Gennip, J.K.J.v. Duren, P. Jonkheijm, R.A.J. Janssen, J.W. Niemantsverdriet, Appl. Surf. Sci., 203-204 (2003) 547-550.

[51] J.A. Emerson, D.T.W. Toolan, J.R. Howse, E.M. Furst, I. Thomas H. Epps, Macromolecules, 46 (2013) 6533-6540.

[52] S.M. Naga Rajesh Tummala, Yao-Tsung Fu, Chad Risko, Jean-Luc Bredas, Advance Functional Materials, 23 (2013) 5800-5813. 
Table 1. Experimental and simulated densities of P3HT and PCBM

\begin{tabular}{lcc}
\hline & Density of P3HT $\left(\mathrm{g} / \mathrm{cm}^{3}\right)$ & Density of PCBM $\left(\mathrm{g} / \mathrm{cm}^{3}\right)$ \\
\hline Experiment & 0.936 & $1.50[50]$ \\
Full-atomistic model & 0.710 & 1.46 \\
Coarse-grained model & 0.822 & 1.47 \\
\hline
\end{tabular}

Table 2. Experimental and simulated solubility parameters of P3HT and PCBM

\begin{tabular}{ccc}
\hline & P3HT $\left((\mathrm{MPa})^{1 / 2}\right)$ & PCBM $\left((\mathrm{MPa})^{1 / 2}\right)$ \\
\hline Experiment & $12.8-20[51]$ & $5.2[52]$ \\
Atomistic model & 28.9 & 8.01 \\
Coarse-grained model & 11.7 & 5.7 \\
\hline
\end{tabular}

Table 3. The coordination number (CN) of $B$ for $A$ of each $A-B$ pair

\begin{tabular}{cccc}
\hline \multirow{2}{*}{ Blend } & \multicolumn{4}{c}{ A-B Pair } \\
\cline { 2 - 4 } & thiophene-thiophene pair & $\mathrm{C}_{60}$-C 60 pair & C $_{60}$-thiophene pair \\
\hline P3HT-PCBM & 2.34 & 1.61 & 5.23 \\
P3NT-PCBM & 2.22 & 1.45 & 3.07 \\
P3DT-PCBM & 2.18 & 1.40 & 2.55 \\
\hline
\end{tabular}




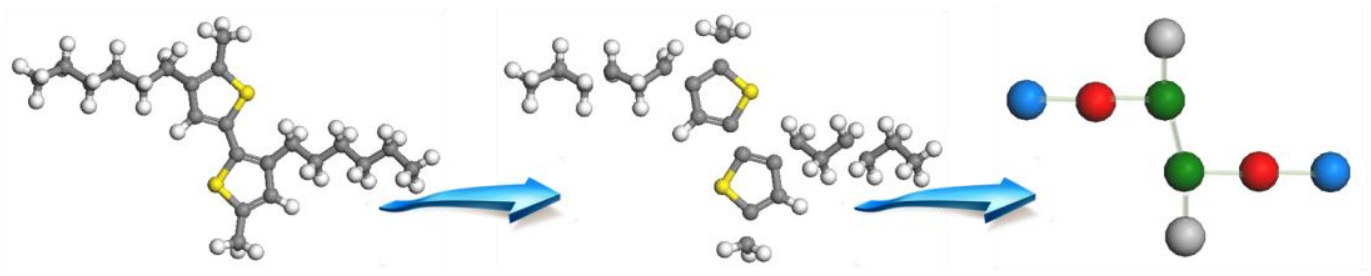

Atomistic model of 3-hexylthiophene dimer

Coarse-grained model of 3-hexylthiophene dimer

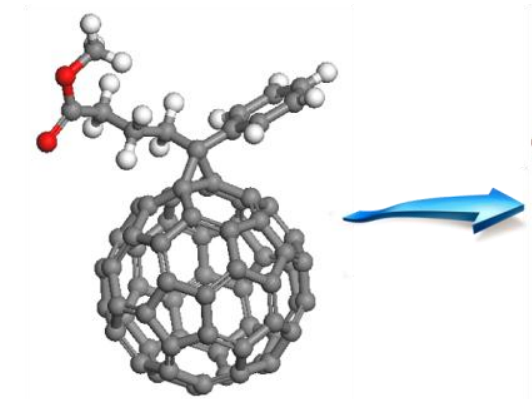

Atomistic model of PCBM

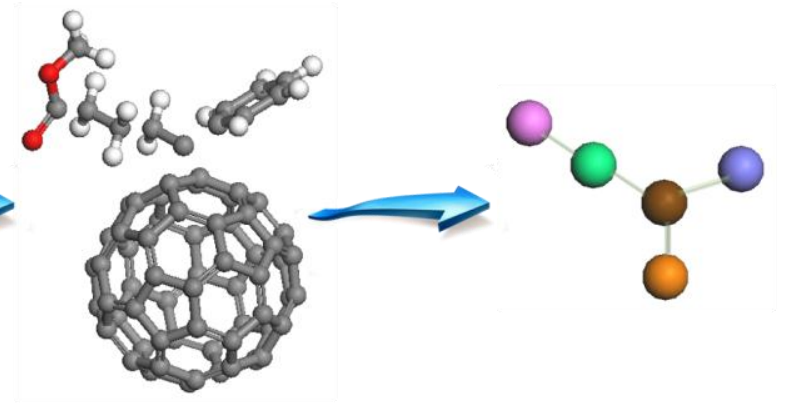

Coarse-grained model of PCBM

Fig. 1. Atomistic and coarse-grained models of 3-hexylthiophene dimer and PCBM. Hereafter, the gray, white, red, and yellow colors depict carbon, hydrogen, oxygen, and sulfur, respectively. 

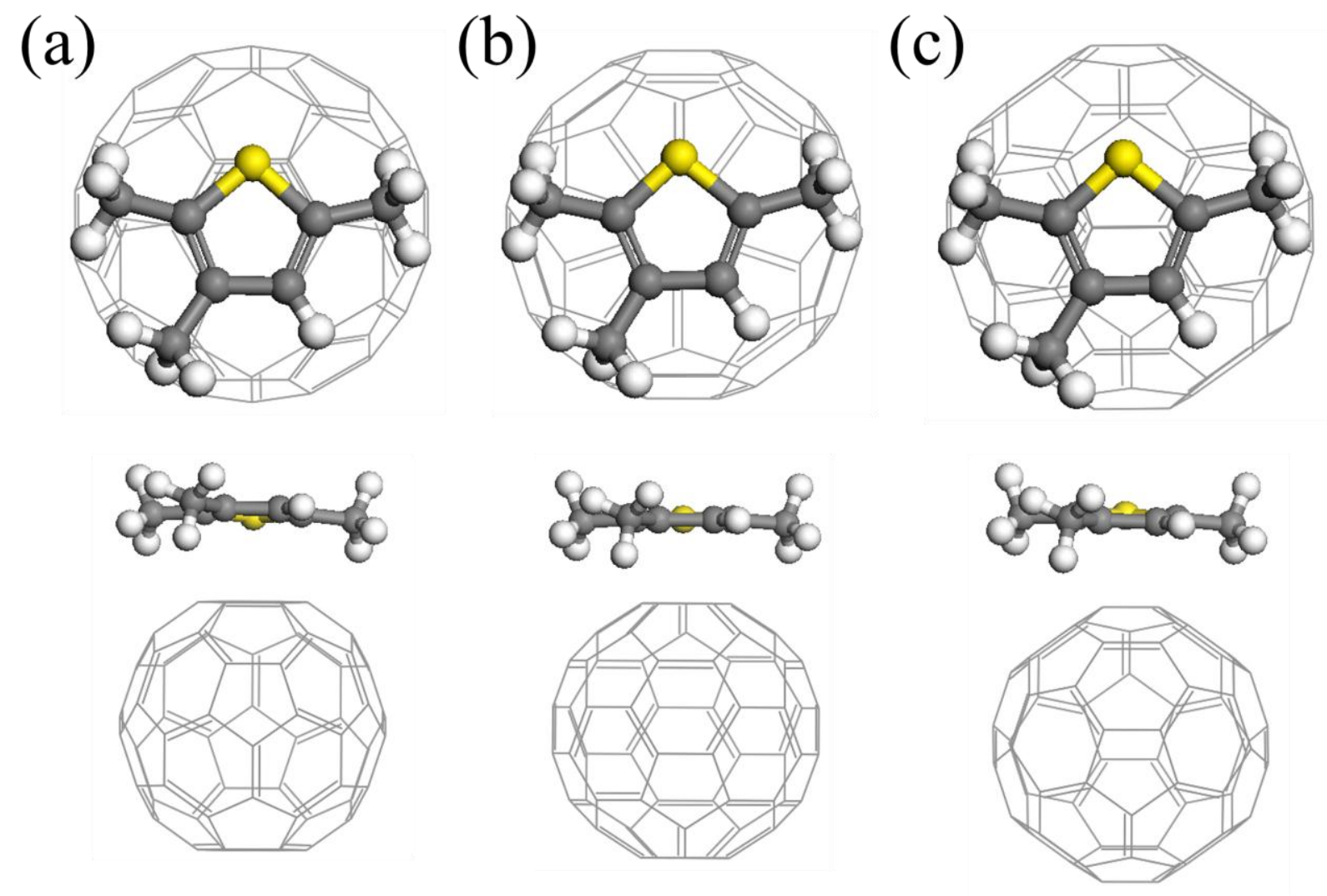

Fig. 2. Top and side views for three configurations of thiophene: $\mathrm{C}_{60}$ atomistic model. Thiophene monomer is on top of (a) a hexagon ring of $\mathrm{C}_{60}$; (b) a pentagon ring of $\mathrm{C}_{60}$; (c) a bond between hexagon and pentagon of $\mathrm{C}_{60}$. 

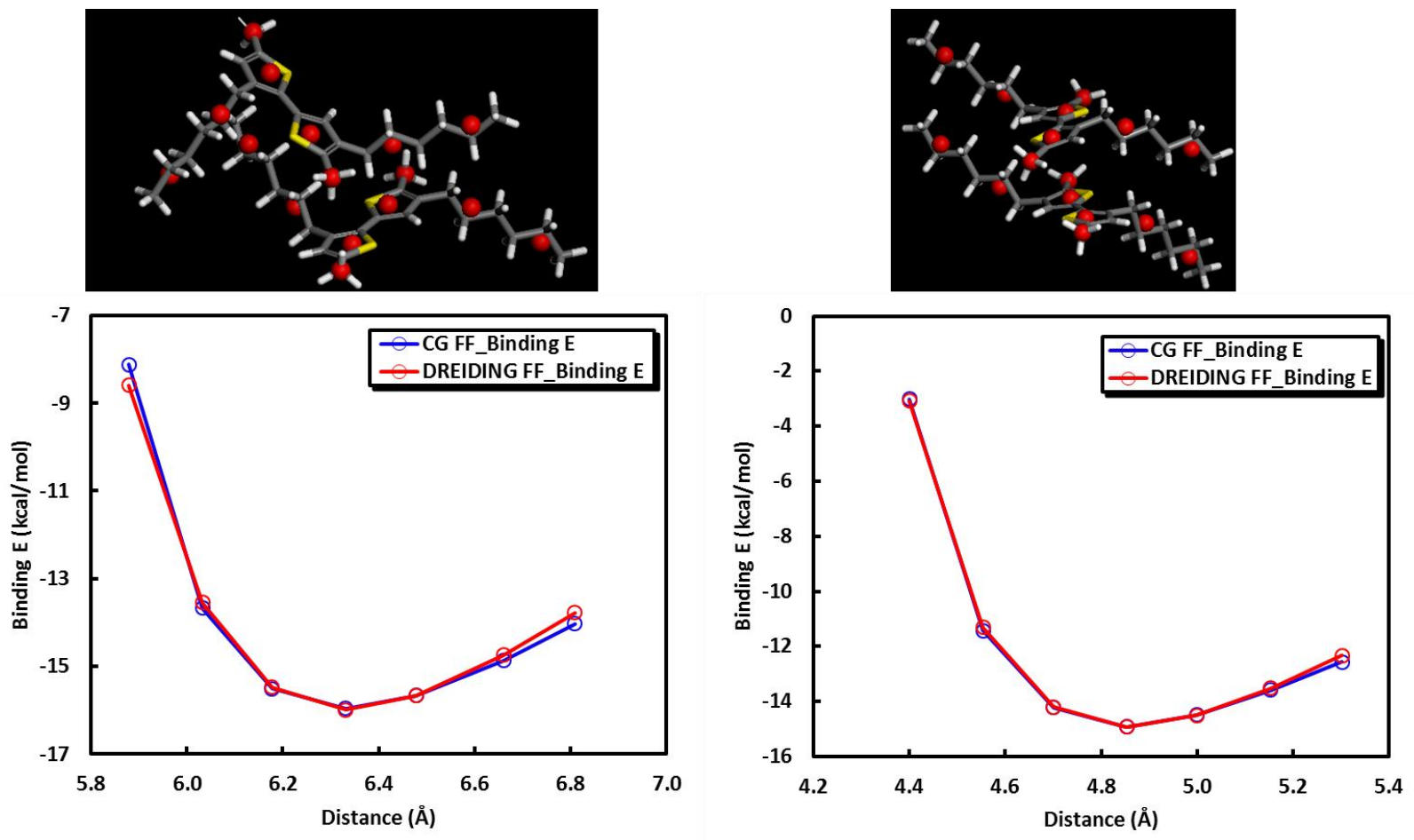

Fig. 3. The potential energy surfaces for the CG P3HT-P3HT pairs. The beads in red represent CG beads. The distance in $x$ axis describes the center of mass (COM) distance between the two P3HTs. 

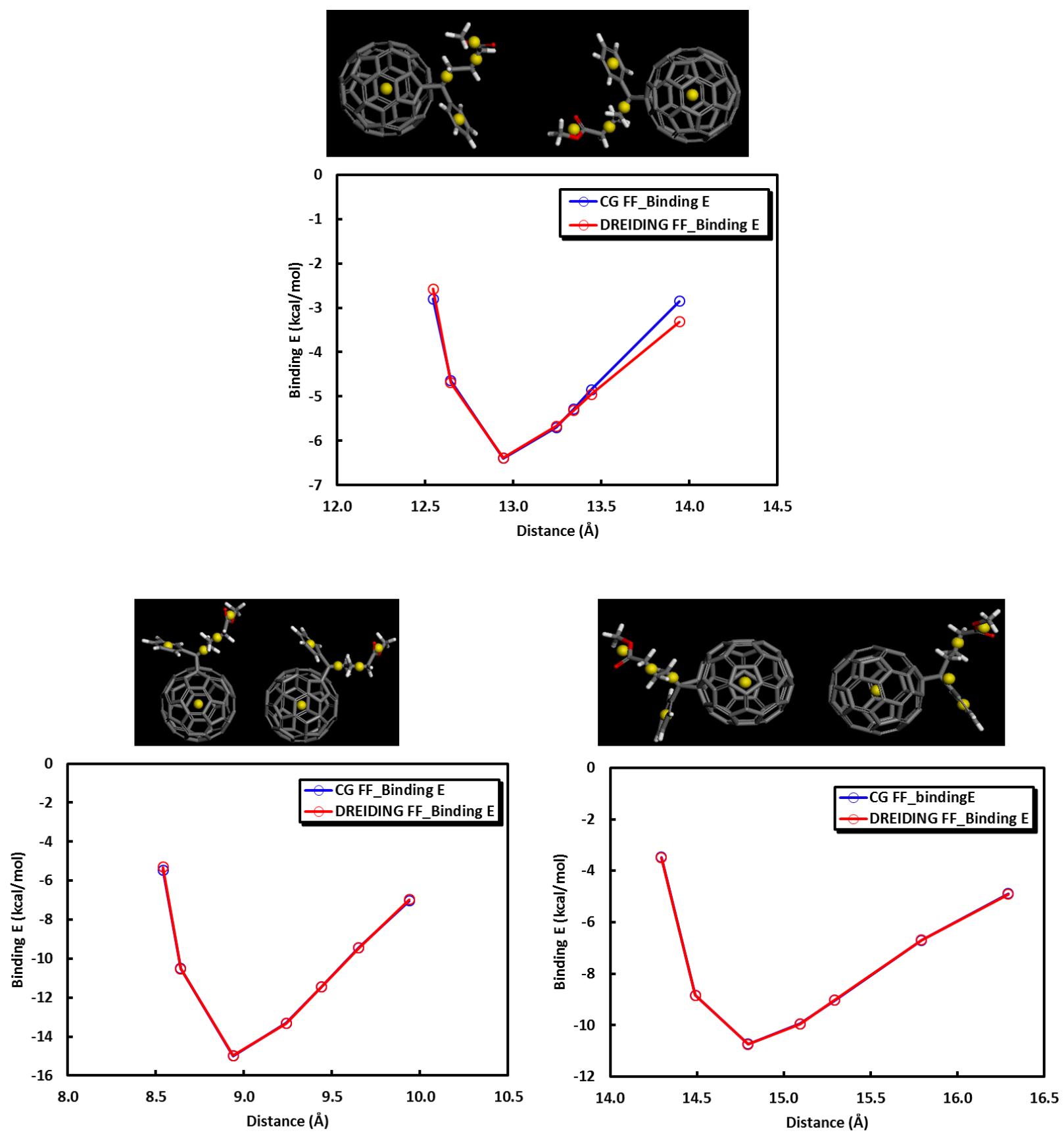

Fig. 4. The potential energy surfaces for the CG PCBM-PCBM pairs. The beads in yellow represent CG beads. The distance in $x$ axis describes the COM distance between the two PCBMs. 


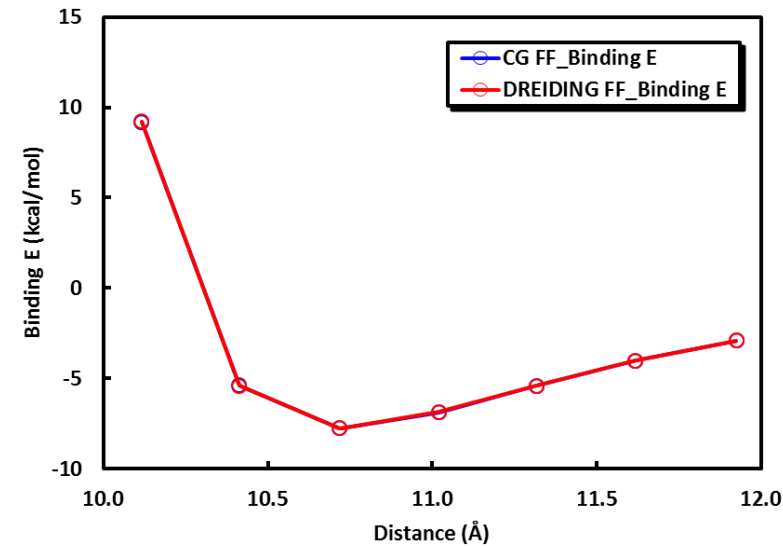

The case of the thiophene in P3HT facing with the functional group in PCBM

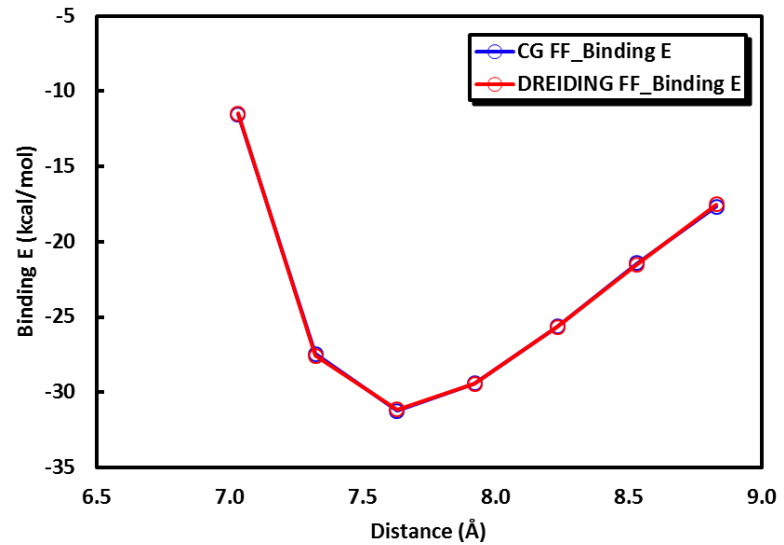

The case of the thiophene in P3HT facing with C60 in PCBM

Fig. 5. The potential energy surfaces for the CG P3HT-PCBM pairs. The distance in $x$ axis describes the COM distance between the P3HT and PCBM. 


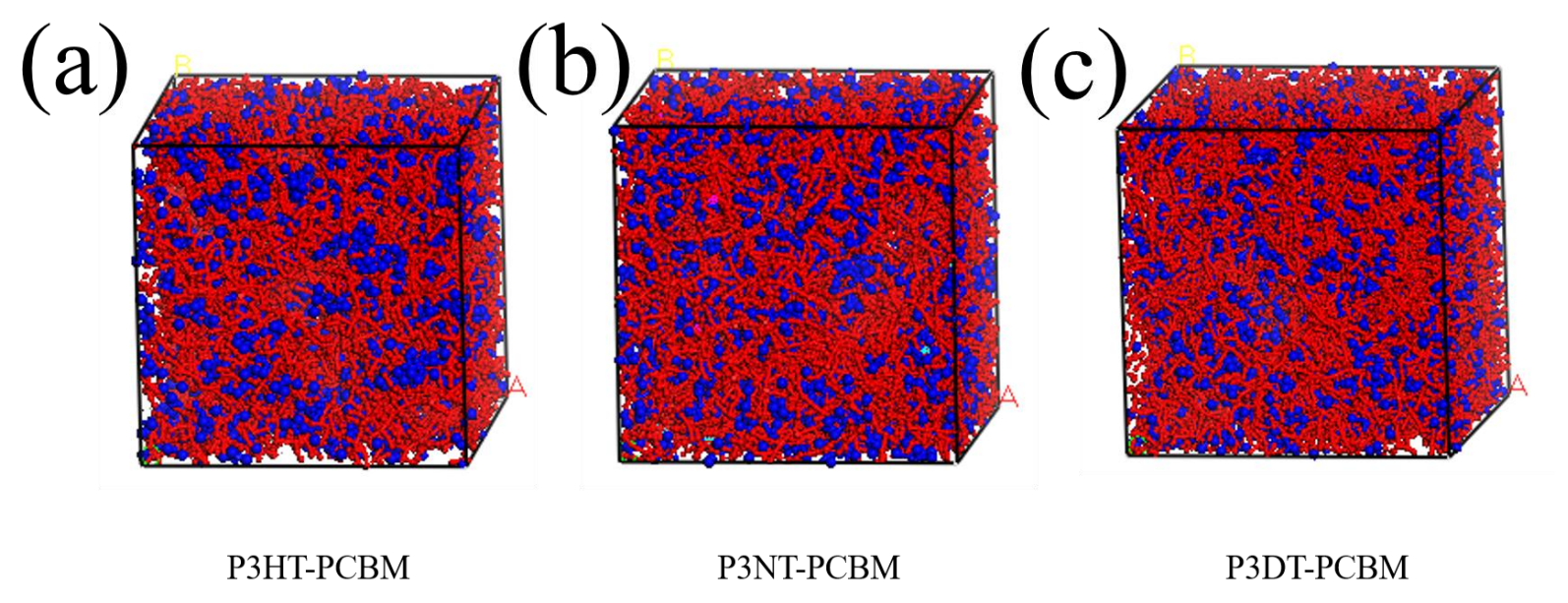

Fig. 6. The equilibrated structures of CG models: (a) P3HT-PCBM, (b) P3NT-PCBM, and (c) P3DTPCBM. The red and blue colors indicate P3AT (A = Hexyl, Nonyl, and Dodecyl) and PCBM, respectively. 


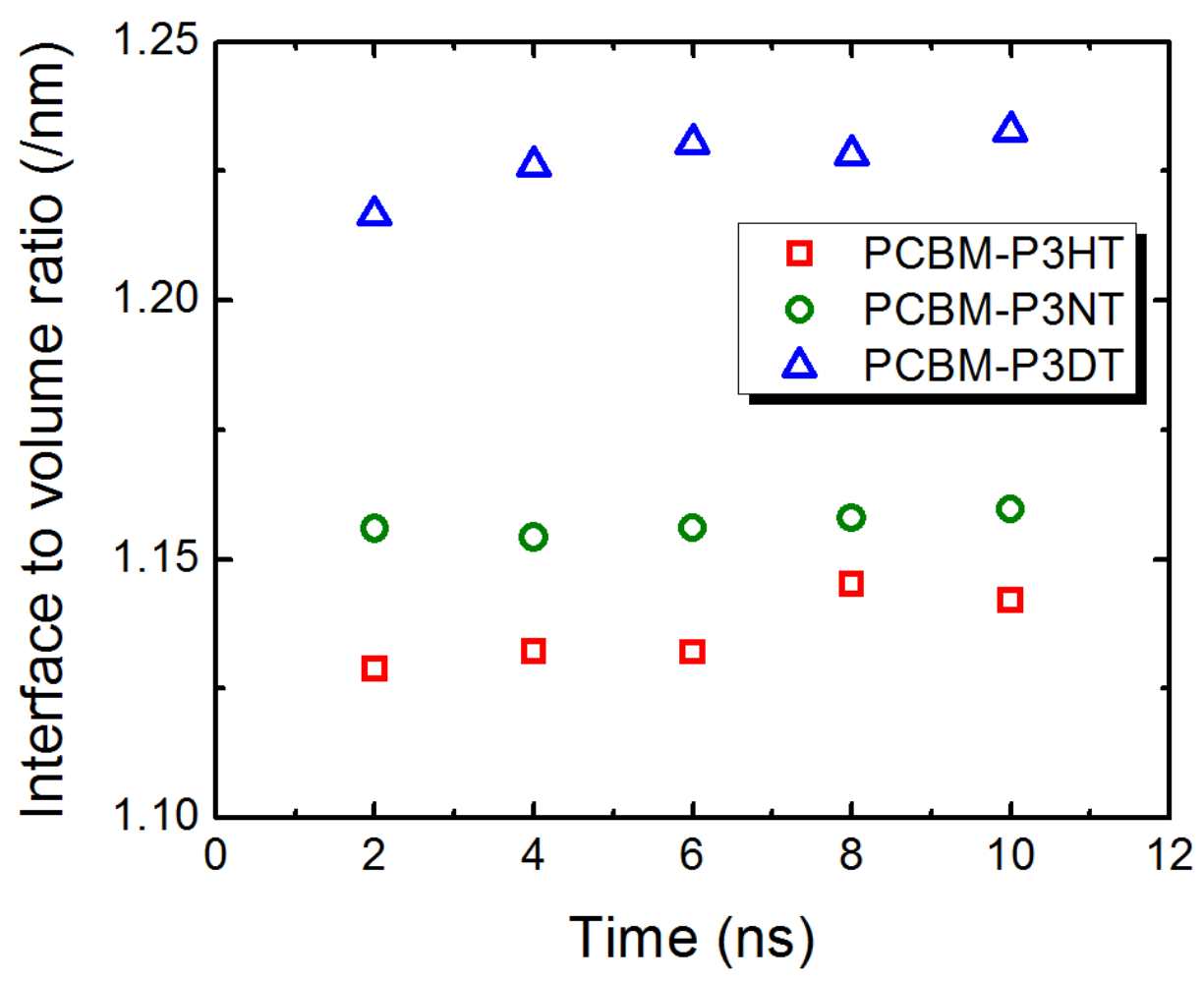

Fig. 7. The change in the interface-to-volume ratio over the simulation time for each of the three active layer models. 

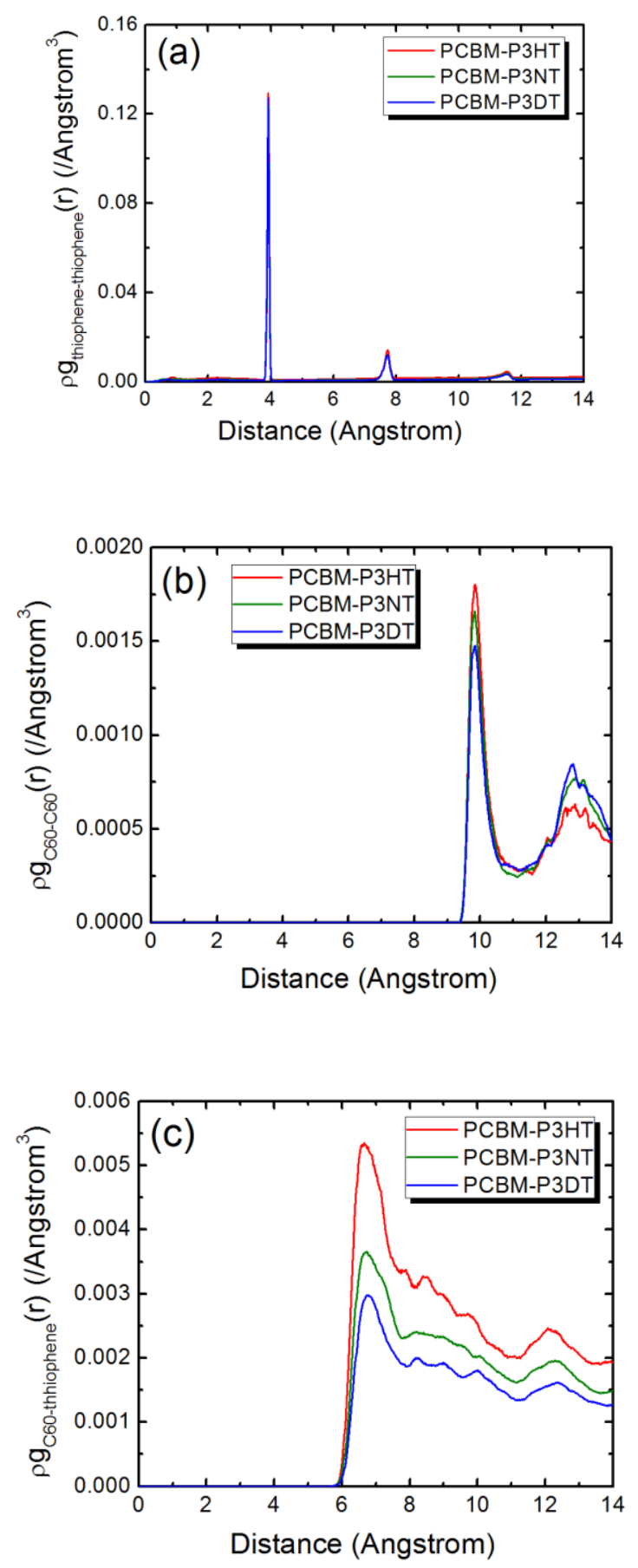

Fig. 8. The number density-weighted pair correlation functions (PCFs) for (a) thiophene-thiophene, (b) $\mathrm{C}_{60}-\mathrm{C}_{60}$, and (c) $\mathrm{C}_{60}$-thiophene $\mathrm{CG}$ pairs. 


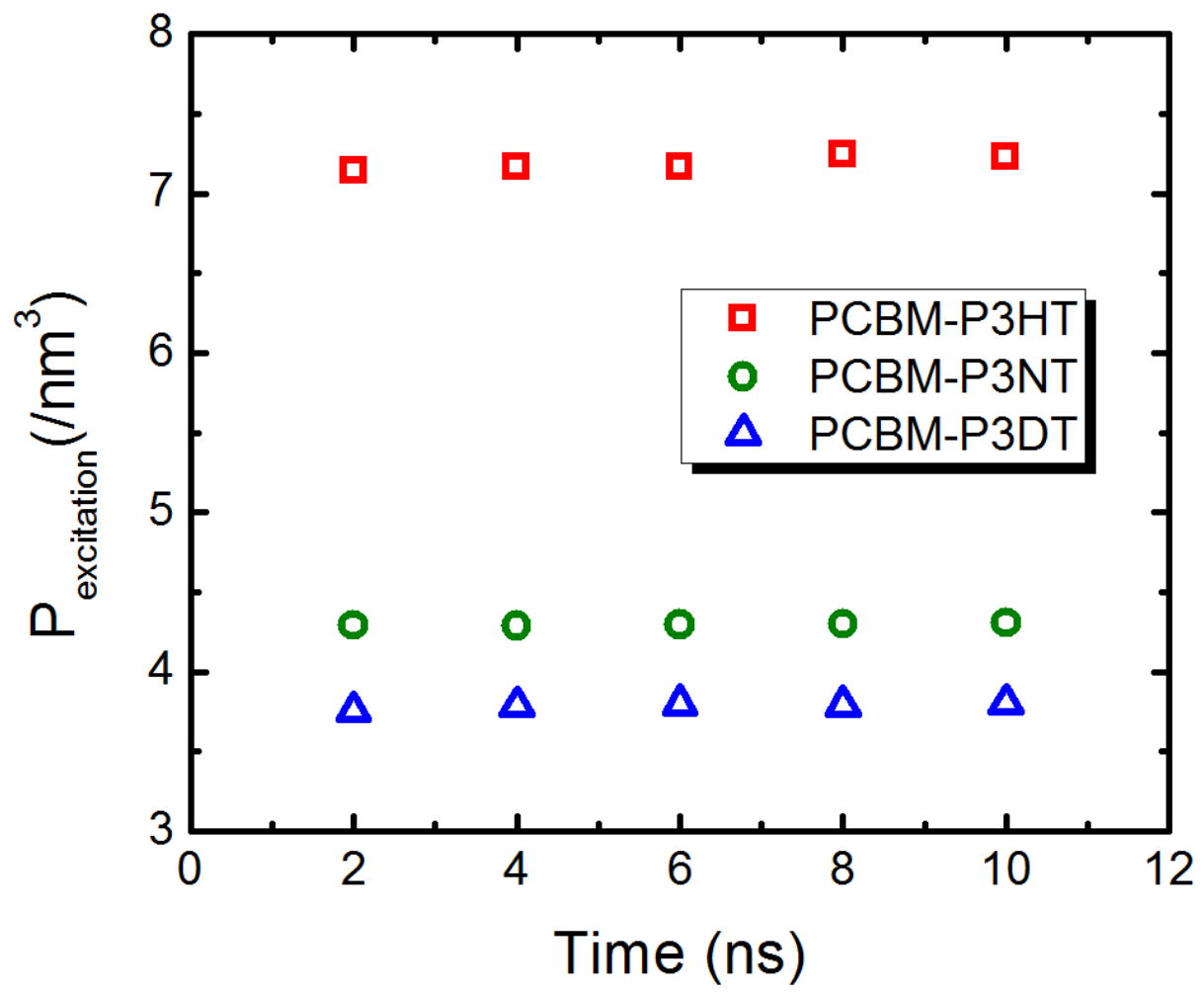

Fig. 9. The profile of $P_{\text {exciton }}$ describing the electron flow from thiophene to C60 through the interface between the electron donor - acceptor for each of the three P3AT-PCBM CG systems. 
Graphic Abstract

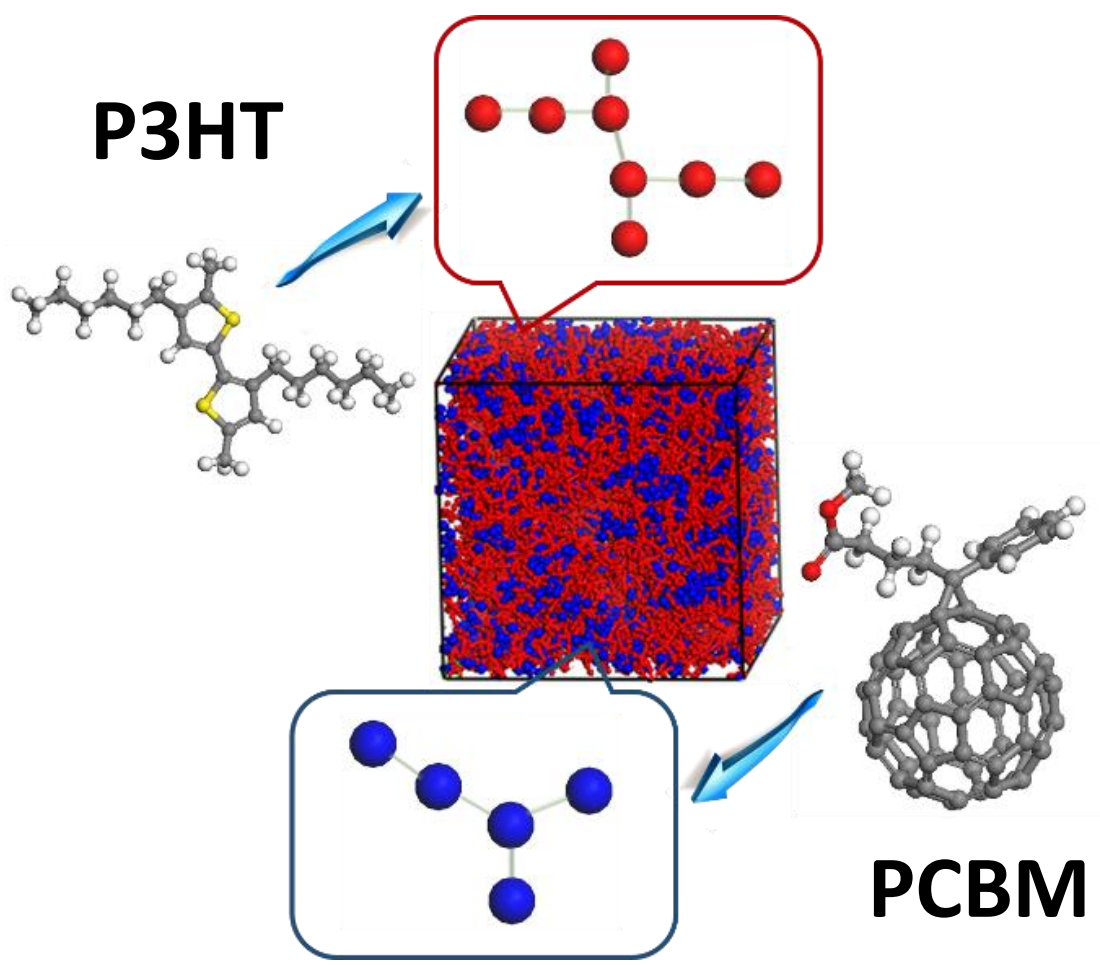

